\title{
The cystic fibrosis airway milieu enhances rescue of F508del in a pre-clinical model
}

\author{
To the Editor:
}

Cystic fibrosis is a life-shortening genetic disease with autosomal recessive inheritance. Most cystic fibrosis morbidity and mortality is associated with pulmonary disease. The cystic fibrosis transmembrane conductance regulator (CFTR) gene, which codes for a chloride channel required for proper hydration of airway epithelial surfaces, is mutated in cystic fibrosis, resulting in airway dehydration. As a result, cystic fibrosis patients suffer from chronic airway infection, inflammation and overproduction of mucus, which leads to airway obstruction. During the last decade, basic and clinical research has led to strategies for targeted therapies to successfully restore CFTR function in cystic fibrosis patients.

The most common mutated protein in cystic fibrosis, F508del CFTR, is retained in the endoplasmic reticulum, which prevents significant amounts of the protein to reach the apical surface of epithelial cells. Because efficient correction of F508del CFTR should benefit most cystic fibrosis patients, it has a high priority in cystic fibrosis therapy. In 2015, the US Food and Drug Administration approved Orkambi, consisting of the corrector lumacaftor (VX-809) plus the potentiator ivacaftor (VX-770), for F508del homozygous patients. Phase 2 and 3 clinical trials of Orkambi for these cystic fibrosis patients demonstrated only modest improvements in lung function $[1,2]$ similar in magnitude to conventional therapies for downstream pathophysiology, e.g. dornase alfa, hypertonic saline or azithromycin [3]. Comparable outcomes were obtained with the new drug, Symdeko, consisting of the corrector tezacaftor (VX-661) in combination with VX-770 [4]. Hence, there is an unmet need to improve the in vivo effectiveness of CFTR-targeting treatments for F508del homozygous patients.

CFTR correctors have not been investigated in vitro under the inflammatory conditions found in cystic fibrosis airways in vivo. Because inflammation of human bronchial epithelia (HBE) increases the endoplasmic reticulum protein folding capacity by up-regulating the expression of endoplasmic reticulum chaperone proteins, folding enzymes and lipids [5-7], we hypothesised that inflammation of HBE would enhance the correcting effect of VX-809 and, thus, the therapeutic benefit of VX-809 and VX-770 in vitro.

To evaluate the action of VX-809 and VX-770 under conditions that recapitulate the inflammatory status of cystic fibrosis airways, we utilised a pre-clinical model consisting of long-term (30-40 day old) cultured, well-differentiated F508del/F508del HBE grown at the air-liquid interface [6] and exposed to supernatant from mucopurulent material (SMM) harvested from the airways of excised human cystic fibrosis lungs [5-8]. SMM contains the soluble infectious and inflammatory factors present in cystic fibrosis airways in vivo [8], such as bacterial products, neutrophil factors, macrophage and epithelial cytokines, mucins and hundreds of peptides. Its cytokine composition is fairly reproducible from patient to patient [8]. Because the airway epithelia of cystic fibrosis patients are exposed to the combination of all the factors present in SMM, as opposed to a single inflammatory factor, we reasoned that the use of SMM (versus the use of a defined stimulus) would be more relevant to evaluate the impact of the cystic fibrosis airway inflammatory milieu on the efficacy of CFTR modulators. In this study, SMM was pooled from eight individual human cystic fibrosis lungs.

We utilised a protocol that combines $24 \mathrm{~h}$ treatment with VX-809 and acute treatment with VX-770 to provide conditions that maximally enhance CFTR function. This protocol is currently most commonly

@ERSpublications

The CF airway inflammatory milieu has a major impact on the efficacy of CFTR modulators, indicating that pre-clinical evaluation under conditions that mimic native inflamed $\mathrm{CF}$ airways is critical for optimising therapies. http://ow.ly/VrD130m770X

Cite this article as: Gentzsch M, Cholon DM, Quinney NL, et al. The cystic fibrosis airway milieu enhances rescue of F508del in a pre-clinical model. Eur Respir J 2018; 52: 1801133 [https://doi.org/ 10.1183/13993003.01133-2018]. 
used in studies on HBE cells conducted by research groups in industry and academia. Well-differentiated F508del/F508del HBE cultures were exposed for $24 \mathrm{~h}$ to apically added PBS or SMM [5-8] in combination with basolateral exposure to vehicle or $5 \mu \mathrm{M}$ VX-809. Notably, long-term cystic fibrosis cultures do not exhibit a hyperinflammatory phenotype in the absence of SMM [6]. Subsequently, analyses of CFTR were performed based on a) functional ion transport studies utilising an Ussing chamber protocol that allows sensitive measurements of endogenous F508del activity, which included acute addition of forskolin, VX-770 and CFTR inhibitor (CFTRinh-172), b) biochemical evaluation of protein maturation by Western blots [9-11], and c) evaluation of CFTR mRNA levels by quantitative reverse transcriptase polymerase chain reaction (RT-PCR), utilising our established method [8].

Figure 1a illustrates that VX-809-treated F508del/F508del HBE cultures exhibited a larger CFTR-mediated response to forskolin and VX-770 versus vehicle-treated cultures. Surprisingly, these responses were drastically enhanced by the SMM treatment (figure 1a). VX-809-increased forskolin+VX-770-induced $\mathrm{Cl}^{-}$ secretory responses were enhanced by SMM exposure in a dose-dependent manner (data not shown). Notably, SMM-enhanced VX-809-increased forskolin+VX-770 responses were blunted by CFTRinh-172 (figure 1a). The compiled data for the quantification of forskolin+VX-770-induced F508del-mediated responses and inhibition by CFTRinh-172 are shown in figure $1 \mathrm{~b}$.

To address whether SMM and/or VX-809 treatment affected additional bioelectric responses beyond those mediated by CFTR, we evaluated the baseline currents, amiloride responses, residual/amiloride-insensitive currents and UTP responses in all groups. The compiled data from these analyses are depicted in figure 1c. Although baseline short-circuit currents $\left(\mathrm{Isc}_{\mathrm{sc}}\right)$ were not significantly different in any group, there was a trend for basal currents to be higher in SMM-treated cultures. In addition, there was a trend for amiloride responses to be reduced in the VX-809-treated groups, presumably due to restoration of CFTR function and subsequent inhibition of activity of the epithelial sodium channel, ENaC. Residual currents were slightly enhanced in the SMM-treated groups, which may be due to increased baseline function of CFTR (prior to forskolin activation) and/or activation of additional channels, e.g., $\mathrm{Ca}^{2+}$-activated $\mathrm{Cl}^{-}$channels (CaCC). Indeed, as previously described [8], UTP responses were enhanced in SMM-treated cultures, possibly reflecting an up-regulation of $\mathrm{CaCC}$ function. Moreover, we cannot rule out direct or indirect contributions of other channels that may be affected by SMM. Nevertheless, because rescued CFTR responses were the predominant currents affected by SMM and largely inhibited by CFTRinh-172, our data indicate the existence of a high selectivity and specificity for the action of SMM on repaired F508del versus other channels.

In agreement with the functional data, Western blot analyses indicated that mainly the immature endoplasmic reticulum-residing form of CFTR (band B) was detected in the absence of VX-809 (figure 1d). Notably, while band B was not significantly increased in cultures treated with either SMM or VX-809, it was considerably increased in cultures simultaneously treated with SMM and VX-809 (figure 1d and e, $\mathrm{SMM} / \mathrm{VX}-809)$. On the other hand, while the mature band C was formed in F508del cultures treated only with VX-809 (PBS/VX-809), the SMM treatment further increased the amount of VX-809-rescued mature band C (figure 1d and f, SMM/VX-809). Utilising the same experimental conditions, we found that SMM exposure did not significantly increase the mRNA levels of CFTR in the presence or absence of VX-809 (figure 1g). The findings from figure $1 \mathrm{~d}$, e and g suggest that SMM does not significantly increase transcription or translation of F508del. Rather, our data lead to the notion that the mechanism for SMM-enhanced CFTR levels in the presence of VX-809 may be mediated by SMM-induced F508del stabilisation, resulting in decreased CFTR protein degradation. Indeed, preliminary data from our laboratories suggest that SMM overcomes destabilisation of VX-809-rescued F508del by chronic VX-770 treatment in vitro (data not shown).

Contrary to our findings, previous studies suggested that Pseudomonas aeruginosa reduces VX-809- and VX-809+VX-770-stimulated F508del CFTR-mediated $\mathrm{Cl}^{-}$secretion [12]. However, there are possible explanations for this discrepancy. First, because these studies were conducted $6 \mathrm{~h}$ after Pseudomonas aeruginosa exposure, we speculate that the time of bacterial exposure might not have been sufficient to expand the endoplasmic reticulum compartment and increase its protein folding capacity, as we have reported [5], to enhance VX-809-mediated F508del rescue. Second, because Pseudomonas aeruginosa constitutes a single stimulus that does not recapitulate all the infectious/inflammatory factors present in cystic fibrosis airways, unlike SMM, it does not likely trigger activation of many signalling pathways necessary to efficiently promote endoplasmic reticulum expansion in HBE in vitro.

Our findings provide a new model for pre-clinical evaluation of CFTR modulators. There are very little data regarding the impact of inflammation on the efficacy of CFTR modulators in human subjects. However, because increased inflammation occurs very early in cystic fibrosis lung disease, the airway inflammation present even in mild disease may be sufficient to trigger the effects noted in this study. The 
a)
PBS/Vehicle
SMM/Vehicle
PBS/VX-809
SMM/VX-809
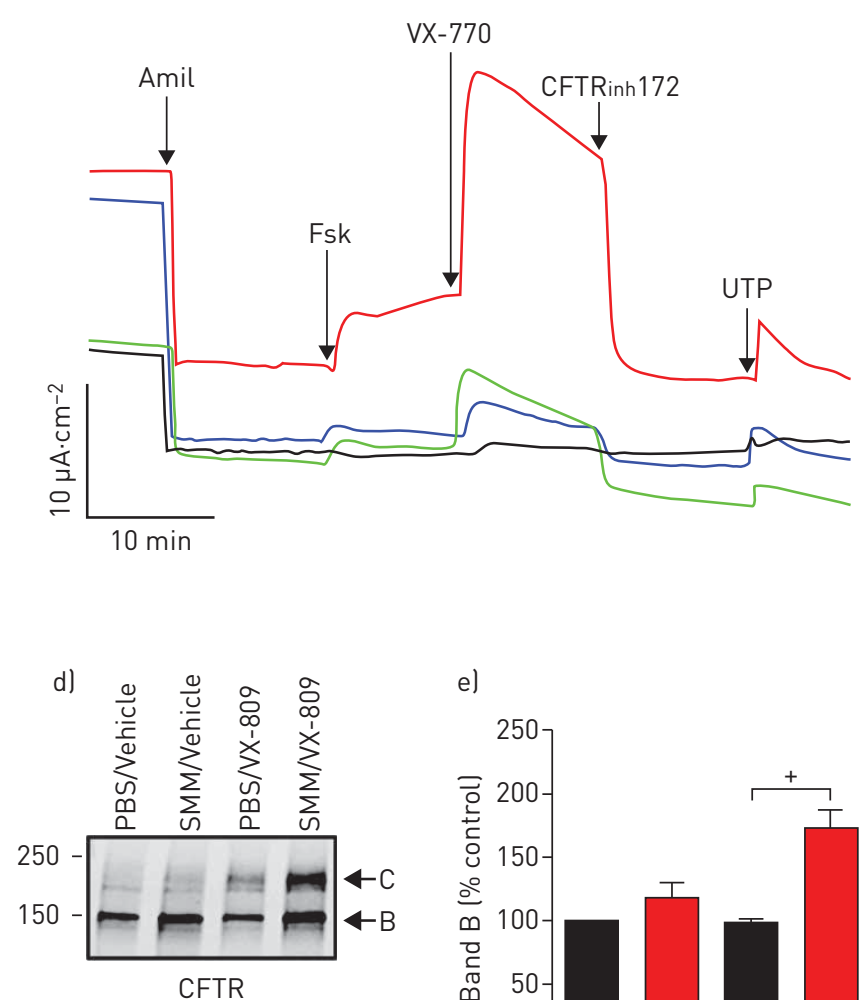

50

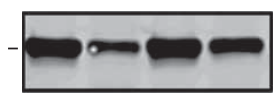

Tubulin b)

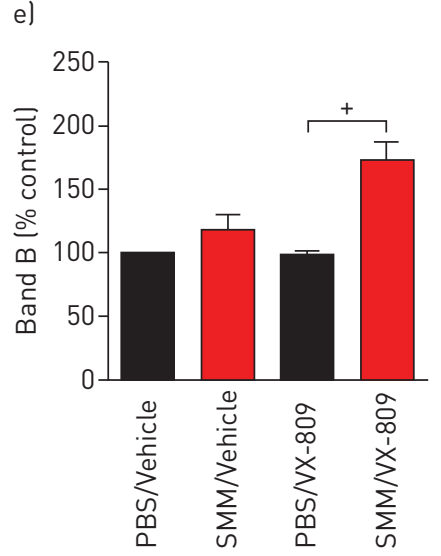

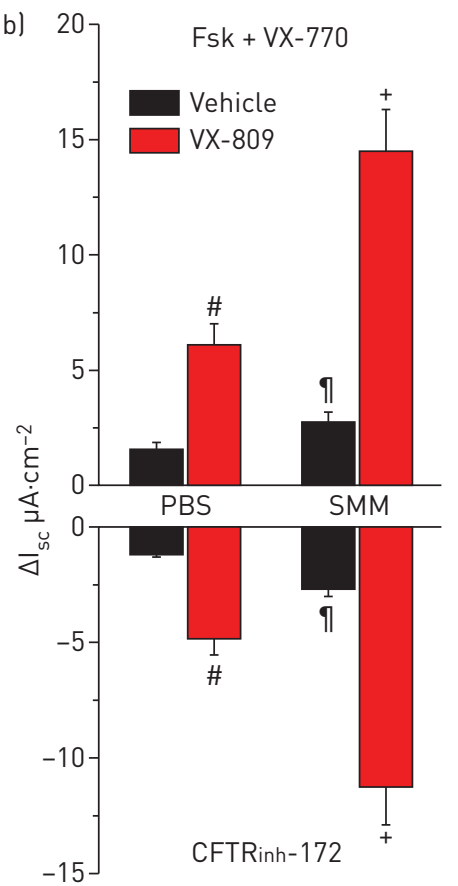

f)

c)

\begin{tabular}{|cc|}
\hline Baseline Isc & $\mu \mathrm{A} \cdot \mathrm{cm}^{-2}$ \\
\hline PBS/Vehicle & $23.23 \pm 3.87$ \\
\hline SMM/Vehicle & $30.90 \pm 2.64$ \\
\hline PBS/VX-809 & $20.68 \pm 2.86$ \\
\hline SMM/VX-809 & $24.60 \pm 2.00$ \\
\hline Amiloride & $\mu \mathrm{A} \cdot \mathrm{cm}^{-2}$ \\
\hline PBS/Vehicle & $-22.41 \pm 4.49$ \\
\hline SMM/Vehicle & $-26.77 \pm 2.34$ \\
\hline PBS/VX-809 & $-18.23 \pm 3.60$ \\
\hline SMM/VX-809 & $-20.70 \pm 2.09$ \\
\hline Residual Isc & $\mu \mathrm{A} \cdot \mathrm{cm}^{-2}$ \\
\hline PBS/Vehicle & $0.84 \pm 0.76$ \\
\hline SMM/Vehicle & $4.18 \pm 1.37$ \\
\hline PBS/VX-809 & $2.46 \pm 1.23$ \\
\hline SMM/VX-809 & $3.91 \pm 1.24$ \\
\hline UTP & $\mu \mathrm{A} \cdot \mathrm{cm}^{-2}$ \\
\hline PBS/Vehicle & $0.85 \pm 0.22$ \\
\hline SMM/Vehicle & $3.21 \pm 0.57$ \\
\hline PBS/VX-809 & $1.02 \pm 0.45$ \\
\hline SMM/VX-809 & $2.43 \pm 0.45$ \\
\hline
\end{tabular}

g)

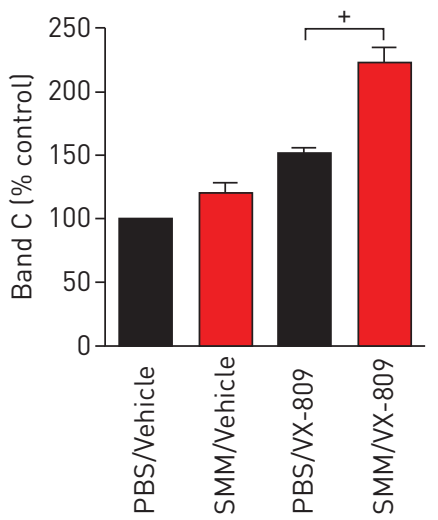

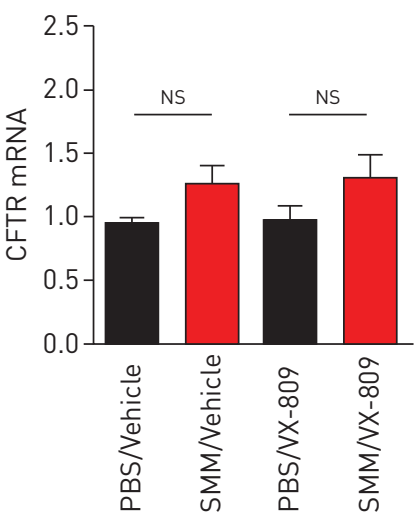

FIGURE 1 SMM exposure enhances functional rescue of F508del. F508del/F508del HBE cultures were exposed for $24 \mathrm{~h}$ to vehicle or $5 \mu \mathrm{M}$ VX-809 in combination with $30 \mu \mathrm{L}$ mucosal PBS or SMM. a) Representative short-circuit currents $\left(\mathrm{I}_{\mathrm{sc}} \mu \mathrm{A} \cdot \mathrm{cm}^{-2}\right)$ recorded in Ussing chambers.

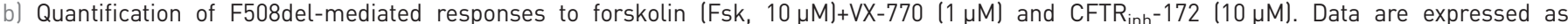
mean \pm SEM. ${ }^{\#}:$ : $p<0.05$ VX-809 versus vehicle; ${ }^{\uparrow}: p<0.05$, SMM versus PBS; ${ }^{+}: p<0.05$, SMM/VX-809 versus PBS/VX-809. $n=4$ F508del/F508del HBE culture codes, and experiments were performed in 3-4 replicates/code in 14 measurements. c) Compiled data from baseline, amiloride-sensitive, residual (amiloride-insensitive) and UTP-dependent Isc responses. d) Representative Western blot of immunoprecipitated CFTR and tubulin controls. e) Quantification of band B as \% values normalised from band B values from control (vehicle- and PBS-treated CF HBE). Data are

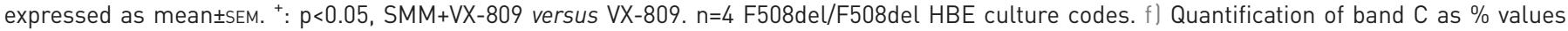
normalised from band $C$ values from control (vehicle- and PBS-treated CF HBE). Data are expressed as mean \pm SEM. ${ }^{+}:$p $<0.05, S M M+V X-809$ versus VX-809. N=4 F508del/F508del HBE culture codes. g) CFTR mRNA levels, determined by RT-PCR. Data are expressed as fold change relative to TATA-box binding protein mRNA. $n=3$ F508del/F508del HBE culture codes.

dose-response relationship between CF airway inflammation and efficacy of CFTR modulators should be addressed in future studies. Comparisons between the airway inflammatory status of CF patients with in vivo efficacy of Orkambi or Symdeko, and studies with cells from these patients exposed to their own airway secretions will address this relationship.

The acquired knowledge from our studies may also be applicable for rescuing rare CFTR mutations [13], offering pre-clinical models and precise treatment strategies for all CF patients. Furthermore, the proposed studies may be relevant to other airway diseases, i.e., COPD, where dysfunctional CFTR is emerging as a therapeutic target [14]. Additional studies will be necessary to identify the mechanism underlying the enhancement of VX-809-mediated F508del rescue during inflammation, which may shed light on the lack of benefit of VX-809 as monotherapy in clinical trials. We speculate that the initial enhancement of VX-809-mediated restoration of CFTR activity under inflammatory conditions is counteracted, with time, 
by its correcting effect (e.g. correction of CFTR function might decrease the airway epithelial inflammatory response, thereby decreasing VX-809 correction). In fact, recent data indicating that restoration of functional CFTR reduces inflammation in cystic fibrosis airways [15] agree with this notion. Notably, the next generation of correctors may have a more significant impact on decreasing airway inflammatory responses of cystic fibrosis patients.

In conclusion, our study indicates that the cystic fibrosis airway infectious/inflammatory milieu has a major impact on the efficacy of CFTR modulators. As more CFTR modulator combinations become available (see www.cff.org/Trials/Pipeline), pre-clinical evaluation of the efficacy of modulators under conditions that mimic native inflamed cystic fibrosis airway epithelia may be critical for optimisation of cystic fibrosis therapies.

Martina Gentzsch ${ }^{1,2}$, Deborah M. Cholon ${ }^{1}$, Nancy L. Quinney ${ }^{1}$, Susan E. Boyles ${ }^{1}$, Mary E.B. Martino ${ }^{1}$ and

${ }^{1}$ Marsico Lung Institute and Cystic Fibrosis Research Center, Chapel Hill, NC, USA. ${ }^{2}$ Dept of Cell Biology and Physiology, The University of North Carolina, Chapel Hill, NC, USA. ${ }^{3}$ Dept of Medicine, The University of North Carolina, Chapel Hill, NC, USA.

Correspondence: Martina Gentzsch, University of North Carolina at Chapel Hill, School of Medicine - Cell Biology and Physiology, 1121 Marsico Hall CB 7248, 125 Mason Farm Road, Chapel Hill, NC 27599, USA. E-mail: martina_gentzsch@med.unc.edu

Received: June 152018 | Accepted after revision: Sept 222018

Acknowledgements: The authors thank Scott H. Randell and the University of North Carolina Cystic Fibrosis Center Tissue Procurement and Cell Culture Core for providing HBE cells and mucopurulent material from human CF airways (supported by Cystic Fibrosis Foundation grant R026-CR11 and NIH grant P30DK065988), and Charles R. Esther, Jr, for critical discussion.

Conflict of interest: None declared.

Support statement: This study was supported by the Cystic Fibrosis Foundation (R026-CR11, BOUCHE15R0, RIBEIR17P0), Cystic Fibrosis Research, Inc. (2018NH-001) and the National Institutes of Health (P30DK065988 and R56HL136733). Funding information for this article has been deposited with the Crossref Funder Registry.

\section{References}

1 Wainwright CE, Elborn JS, Ramsey BW, et al. Lumacaftor-ivacaftor in patients with cystic fibrosis homozygous for Phe508del CFTR. N Engl J Med 2015; 373: 220-231.

2 Boyle MP, Bell SC, Konstan MW, et al. A CFTR corrector (lumacaftor) and a CFTR potentiator (ivacaftor) for treatment of patients with cystic fibrosis who have a phe508del CFTR mutation: a phase 2 randomised controlled trial. Lancet Respir Med 2014; 2: 527-538.

3 Cholon DM, Esther CR Jr, Gentzsch M. Efficacy of lumacaftor-ivacaftor for the treatment of cystic fibrosis patients homozygous for the F508del-CFTR mutation. Expert Rev Precis Med Drug Dev 2016; 1: 235-243.

4 Taylor-Cousar JL, Munck A, McKone EF, et al. Tezacaftor-ivacaftor in patients with cystic fibrosis homozygous for Phe508del. N Engl J Med 2017; 377: 2013-2023.

5 Martino ME, Olsen JC, Fulcher NB, et al. Airway epithelial inflammation-induced endoplasmic reticulum Ca2+ store expansion is mediated by X-box binding protein-1. J Biol Chem 2009; 284: 14904-14913.

6 Ribeiro CM, Paradiso AM, Schwab U, et al. Chronic airway infection/inflammation induces a Ca2+i-dependent hyperinflammatory response in human cystic fibrosis airway epithelia. J Biol Chem 2005; 280: 17798-17806.

7 Ribeiro $\mathrm{CM}$, Hurd $\mathrm{H}, \mathrm{Wu} \mathrm{Y}$, et al. Azithromycin treatment alters gene expression in inflammatory, lipid metabolism, and cell cycle pathways in well-differentiated human airway epithelia. PLoS ONE 2009; 4: e5806.

8 Abdullah LH, Coakley R, Webster MJ, et al. Mucin production and hydration responses to mucopurulent materials in normal versus cystic fibrosis airway epithelia. Am J Respir Crit Care Med 2018; 197: 481-491.

9 Cholon DM, Quinney NL, Fulcher ML, et al. Potentiator ivacaftor abrogates pharmacological correction of DeltaF508 CFTR in cystic fibrosis. Sci Transl Med 2014; 6: 246 ra296.

10 Gentzsch M, Ren HY, Houck SA, et al. Restoration of R117H CFTR folding and function in human airway cells through combination treatment with VX-809 and VX-770. Am J Physiol Lung Cell Mol Physiol 2016; 311: L550-L559.

11 Gentzsch M, Boyles SE, Cheluvaraju C, et al. Pharmacological rescue of conditionally reprogrammed cystic fibrosis bronchial epithelial cells. Am J Respir Cell Mol Biol 2017; 56: 568-574.

12 Stanton BA, Coutermarsh B, Barnaby R, et al. Pseudomonas aeruginosa reduces VX-809 stimulated F508del-CFTR chloride secretion by airway epithelial cells. PLoS ONE 2015; 10: e0127742.

13 Veit G, Avramescu RG, Chiang AN, et al. From CFTR biology toward combinatorial pharmacotherapy: expanded classification of cystic fibrosis mutations. Mol Biol Cell 2016; 27: 424-433.

14 Solomon GM, Raju SV, Dransfield MT, et al. Therapeutic approaches to acquired cystic fibrosis transmembrane conductance regulator dysfunction in chronic bronchitis. Ann Am Thorac Soc 2016; 13: Suppl. 2, S169-S176.

15 Hisert KB, Heltshe SL, Pope C, et al. Restoring cystic fibrosis transmembrane conductance regulator function reduces airway bacteria and inflammation in people with cystic fibrosis and chronic lung infections. Am J Respir Crit Care Med 2017; 195: 1617-1628. 